\title{
Lubricated sliding friction: Role of interfacial fluid slip and surface roughness
}

\author{
C. Rotella ${ }^{1}$, B.N.J. Persson ${ }^{2,3, a}$, M. Scaraggi ${ }^{2,4}$, and P. Mangiagalli ${ }^{1}$ \\ 1 Sanofi, 13, quai Jules Guesde, BP 14-94403, Vitry-sur-Seine Cedex, France \\ 2 PGI-1, FZ Jülich, Jülich, Germany \\ 3 MultiscaleConsulting, Wolfshovener str 2, 52428 Jülich, Germany \\ 4 DII, Università del Salento, Lecce, Italy
}

Received 30 July 2019 and Received in final form 22 December 2019

Published online: 10 February 2020

(C) The Author(s) 2020. This article is published with open access at Springerlink.com

\begin{abstract}
We derive approximate mean field equations for the fluid flow between elastic solids with randomly rough surfaces including interfacial fluid slip and shear thinning. We present numerical results for the fluid flow and friction factors for realistic systems, in particular, we consider the case of an elastic cylinder with random surface roughness in relative sliding contact with a flat rigid (low-energy) countersurface. We present experimental data for the sliding friction between rubber stoppers and glass barrels lubricated with baked-on silicone oil. We find that the frictional shear stress acting in the rubber asperity contact regions is nearly velocity independent for velocities in the $10-1000 \mu \mathrm{m} / \mathrm{s}$ range, and very small $\tau_{\mathrm{f}} \approx 0.04 \mathrm{MPa}$, while for bare glass in silicone oil $\tau_{\mathrm{f}}$ is much larger and velocity dependent.
\end{abstract}

\section{Introduction}

Lubricated sliding friction is an important but very complex topic. The sliding contact of elastic solids with smooth surfaces in viscous fluids with simple rheology is a well-understood problem [1]. However, real solids have surface roughness on many length scales and most fluids exhibit complex rheology when confined at narrow separation between solid surfaces. Recently, theories, based on the multiscale description of the contact dynamics, have been developed for the sliding dynamics between solids with random roughness on many length scales in fluids with simple shear-thinning rheology [2-5].

In most applications it is assumed that the fluid at the surface of a solid "stick" or adhere to the solid so that the fluid and solid velocities coincide on the solid walls. However, if the lateral (atomic) corrugation of the interaction potential between the solid atoms and the fluid molecules is small enough slip can occur at the interface. This has been observed in many experiments by Krim et al. [6-8] for the sliding of inert gas atoms on metal and graphene surfaces. It is also expected for fluids which exhibit a very large contact angle on the solid (strongly hydrophobic systems). Another very important case are polymer fluids such as silicone oils on smooth inert solids. This can be understood from a very simple argument due to de Gennes [9]: If a fluid slips relative to a flat solid

\footnotetext{
a e-mail: b.persson@fz-juelich.de
}

wall, there will be a frictional shear stress acting on the solid wall which, if the corrugated interaction potential is small enough and the temperature high enough, will be proportional to the slip velocity $[10,11]$

$$
\tau_{v}=C v .
$$

The friction factor $C$ will depend on the number of polymer bed units in contact with the solid wall, but this number is (approximately) independent of the polymer chain length. On the other hand, the viscosity of the polymer fluid increases with increasing chain length. In particular, for silicone oil it is possible to change the chain length, and hence the fluid viscosity, over a huge range. If a fluid is sheared (velocity $v_{0}$ ) between two solid walls at the separation $d$, the shear stress $\tau=\eta\left(v_{0}-v\right) / d$, where $v_{0}$ is the velocity of the upper solid surface relative to the lower solid surface (see fig. 1). Here we have assumed that the fluid "sticks" to the upper solid wall. Using that $\tau=\tau_{v}$ gives the slip velocity on the lower solid surface $(z=0)$ :

$$
v=\frac{v_{0}}{1+C d / \eta} .
$$

Since $C$ does not depend on the chain length it follows that for long enough chain length $C d / \eta<1$ and the fluid will slip on the lower solid wall with a velocity of order the velocity of the upper solid wall. The quantity $l=\eta / C$ is denoted the slip length. If $d \gg l$ the slip of the fluid at the solid wall can be neglected resulting in an effective "stick" boundary condition. 


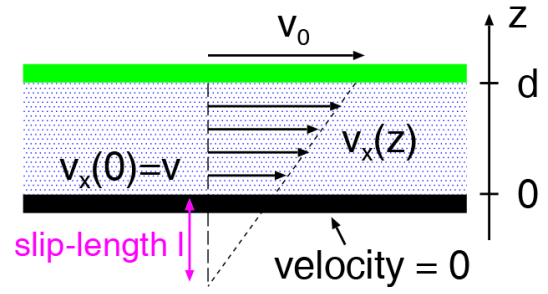

Fig. 1. A fluid sheared between two flat parallel solid walls. The fluid slips relative to the lower solid wall but is assumed to stick to the upper solid wall. The slip length $l$ is the distance below the lower solid surface where the fluid velocity would vanish when extrapolated linearly to negative $z$.

We note that the frictional shear stress $\tau_{v}$ will have the simple form (1) only if the lateral corrugation of the interaction potential between the solid wall atoms and the fluid molecules is small enough so that thermal fluctuations can rapidly transfer molecular segments over the lateral energy barriers. This is typically the case when the interaction potential between the fluid molecules and the solid wall is very weak, and when the fluid molecules form incommensurate-like structures on the solid walls. If this is not the case, the fluid may still slip relative to a solid wall if the viscous shear stress is large enough to overcome the static or pinning shear stress necessary for the initiation of fluid slip relative to the solid wall. In this case the slip length $l$ will depend on the shear velocity $v_{0}$ so that $l \approx 0$ when the fluid shear stress is below some critical value, $\eta v_{0} / d<\tau_{\mathrm{c}}$. In fact, computer simulations of sliding of adsorbed layers [10,11], and experiments for shearing of fluids [12-20], have shown that there may exist several dynamical transitions where the relation $\tau_{v}(v)$, and hence the slip length, changes abruptly with increasing slip velocity $v$. Thus in general we may consider the factor $C$ in (1) to depend on the slip velocity, $C=C(v)$, so the slip length $l=\eta / C(v)$ will depend on the slip velocity. In addition, for most fluids the viscosity $\eta$ will depend on the shear rate, so the slip length will depend on the shear rate (and hence the slip velocity) also via the viscosity $\eta$ term.

In this paper we study the influence of wall fluid slip on the velocity dependence of the friction coefficient, the so called Stribeck curve. We extend the wet contact mechanics theory developed elsewhere $[2,3]$ to include the slip length, and present numerical results for different slip lengths for realistic systems. We consider the case of an elastic cylinder with random surface roughness sliding against a flat rigid (low-energy) counter-surface. This is representative, e.g., of the case of a rubber cylinder sliding against a glass surface with baked-on silicone oil, in a shear-thinning fluid such as silicone oil. We show how the fluid flow and friction factors are affected by the interfacial slip. We also present experimental results for the friction force between the rubber stopper and the glass barrel in syringes, where silicone oil is used as lubricant. The glass barrel has "baked-on" silicone oil and we find that there is fluid slip at the interface between the baked-on film and the silicone oil.

\section{Polymer fluid slip at interfaces}

Most model studies of fluid slip at interfaces have used high-viscosity silicone oils (Polydimethylsiloxan (PDMS) polymer melts). Silicone oils are remarkable fluids with very low surface tension $\left(\gamma \approx 0.02 \mathrm{~J} / \mathrm{m}^{2}\right)$, and with viscosity which can be varied over a huge range, e.g., from $\approx 6 \times 10^{-4} \mathrm{Pas}$ (less than that of water) to $\approx 10^{4} \mathrm{Pas}$ (1000 times higher than the viscosity of honey) by increasing the chain length. Because of its low surface tension, silicone oils wet most surfaces and are excellent lubricant fluids. Silicone oils undergo shear thinning above a critical shear rate which decreases with increasing chain length. The shear thinning involves stretching (aligning) the chain molecules along the shear direction.

In a recent study Henot et al. [21] have presented experimental results for high-viscosity silicone oil in contact with a surface of fused silica covered by end-grafted short PDMS chains, with a molecular weight well below the molecular weight between entanglements. They found that the slip length is proportional to the fluid viscosity as predicted by $l=\eta / C$. This is the expected result when the interaction between the PDMS chains and the solid wall is so weak that the shear stress depends linearly on the slip velocity as in (1). This linear relation is not obeyed if the solid wall is covered by long-chain PDMS molecules which can entangle with the PDMS molecules in the fluid, see fig. 2(a). In this case one observes a transition to a disentangled interface at high enough shear rate (similar to shear thinning). Entanglement can be avoided if the PDMS chains are bounded parallel to the solid wall (see fig. 2(b)), as may be the case for baked-on silicone oil (see below). Another design consists of grafted chain molecules which (energetically) "hate" the molecules in the lubricant fluid so that a phase separation occurs at the sliding interface (see fig. 2(c)) [22].

For PDMS melt against a glass surface coated with a grafted layer of short PDMS chains, Henot et al. found $C \approx 10^{8} \mathrm{Pas} / \mathrm{m}$. Thus for silicone oil with viscosity $\eta=$ 1 Pas we get a slip length $l \approx 10 \mathrm{~nm}$. Another way to passivate glass surfaces is with baked-on silicone oil (see sect. 5). For glass with baked-on silicone oil the PDMS molecules probably bind to the glass surface with the chains partly oriented parallel to the surface. This may result in a friction factor $C$ which may be even smaller than for the end-grafted layer used by Henot et al., resulting in a slip length even larger than $10 \mathrm{~nm}$. Hence, slip at the baked-on silicon oil film may have a large influence on the sliding friction, and could result in a much lower sliding friction force than calculated using the stick boundary condition.

\section{Fluid flow factors with slip}

The Navier-Stokes equations are the basis for fluid dynamics [23]. The fluid flow between narrowly spaced solid walls can usually be described by the Reynolds equations [1] which can be derived from the Navier-Stokes equations assuming that the gap $u(x, y)$ between the solids varies 
(a) entangled fluid and adsorbed film

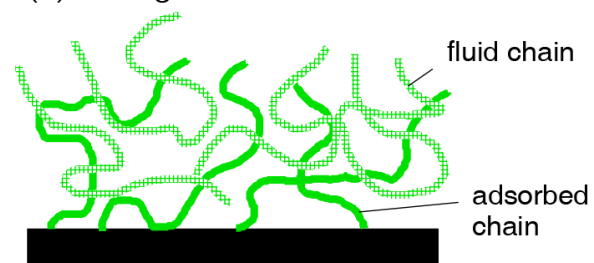

(b) compact adsorbed film: disentangled

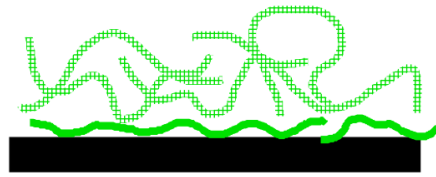

(c) phase separation: disentangled

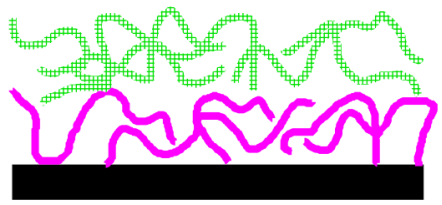

Fig. 2. The interaction between a fluid consisting of long-chain molecules, and a solid surface with adsorbed chain molecules. (a) The adsorbed molecules are of the same type as in the fluid and attached to the wall in one or a few locations, and the rest of the chain are interdiffused with the bulk fluid. (b) The adsorbed chain molecules are of the same type as in the fluid but bound at many locations with the molecule confined tightly to the solid wall (like a snake on the ground). (c) The adsorbed chain molecules (pink) are attached to the wall in one or a few location but energetically do not like the fluid molecules. In this case a phase separation occurs and a well-defined slip plane occurs between the solid and the polymer fluid.

slowly with the lateral $(x, y)$-coordinates. Here we are interested in fluid flow between solids with macroscopically curved surfaces with roughness occurring at length scales much smaller than the radius of curvature $R$ of the macroscopic solids. In this case one can eliminate the surface roughness and obtain effective equations where only the macroscopic variation in the surface profile is explicitly considered (see fig. 3) [2,3,24,25]. The effective equations differ from the Reynolds equation by two functions of the (locally averaged) surface separation $\bar{u}(x, y)$, usually denoted as pressure and shear flow factors, $\phi_{\mathrm{p}}(\bar{u})$ and $\phi_{\mathrm{s}}(\bar{u})$, respectively. In a similar way the frictional shear stress will depend on three functions $\phi_{\mathrm{f}}(\bar{u}), \phi_{\mathrm{fp}}(\bar{u})$ and $\phi_{\mathrm{fs}}(\bar{u})$. In ref. [25] we derived approximate expressions for the fluid and friction factors assuming "stick" boundary conditions. Here we will present a simple (approximate) way to include fluid slip in the theory.

\subsection{Fluid flow current}

Assuming surface roughness with small root mean square (rms) slope, the fluid momentum equation simplifies to

$$
\eta \frac{\partial^{2} \mathbf{v}}{\partial z^{2}}=\nabla p
$$

(a)
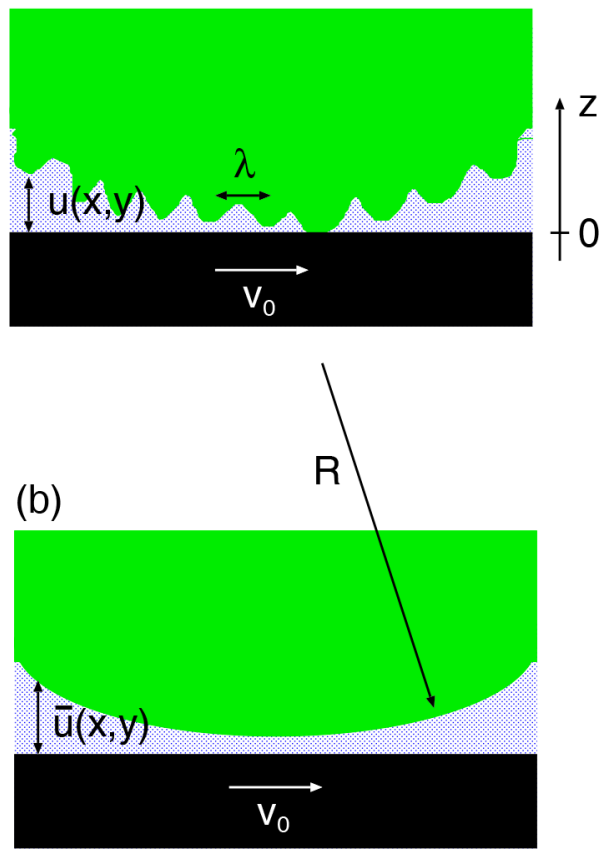

Fig. 3. (a) Elastic block with surface roughness (green) sliding on a flat surface (black). (b) If the macroscopic curvature $R$ of the surfaces of the solids is everywhere much larger than the wavelength $\lambda$ of the most long-wavelength (relevant) surface roughness component, one may "integrate out" (eliminate) the surface roughness and obtain effective fluid flow equations for smooth surfaces. The effective fluid flow equations contain two functions of the average interfacial separation $\bar{u}(x, y)$ which describe the influence of the surface roughness on the fluid flow.

Let us introduce a coordinate system $x y z$ with the $x y$ plane on the surface of the lower solid and with the $z$-axis pointing towards the upper solid (see fig. 3(a)). Hence the fluid velocity field

$$
\mathbf{v}=\frac{1}{2 \eta}\left(z^{2}+a z+b\right) \nabla p+(c+d z) \mathbf{v}_{0}
$$

The boundary condition $\mathbf{v}=\mathbf{0}$ for $z=u(\mathbf{x})$ gives

$$
\mathbf{v}=\frac{1}{2 \eta}\left(z^{2}-u^{2}+a(z-u)\right) \nabla p+d(z-u) \mathbf{v}_{0} .
$$

Next assume that the fluid shear stress acting on the substrate

$$
\sigma_{z x}=C\left[\mathbf{v}_{0}-\mathbf{v}(z=0)\right] .
$$

Using that

$$
\sigma_{z x}=-\eta \frac{\partial \mathbf{v}}{\partial z}
$$

we get

$$
a=-\frac{u^{2}}{u+l}, \quad d=-\frac{1}{u+l}
$$

so that

$$
\mathbf{v}=\frac{1}{2 \eta}\left(z^{2}-u^{2} \frac{z+l}{u+l}\right) \nabla p+\frac{u-z}{u+l} \mathbf{v}_{0} .
$$


The slip length $l=\eta / C$. The fluid flow current

$$
\mathbf{J}=\int_{0}^{u} \mathrm{~d} z \mathbf{v}=-\frac{u^{3}}{12 \eta} \frac{u+4 l}{u+l} \nabla p+\frac{1}{2} \frac{u^{2}}{u+l} \mathbf{v}_{0} .
$$

Here we make the approximation and replace $(u+4 l) /(u+$ 1) with $(\bar{u}+4 l) /(\bar{u}+1)$, where $\bar{u}=\langle u\rangle$ is the ensemble averaged interfacial separation. Performing a similar approximation for $u /(u+l)$ we get

$$
\mathbf{J}=\int_{0}^{u} \mathrm{~d} z \mathbf{v}=-\frac{u^{3}}{12 \eta} \frac{\bar{u}+4 l}{\bar{u}+l} \nabla p+\frac{1}{2} u \frac{\bar{u}}{\bar{u}+l} \mathbf{v}_{0} .
$$

We assume a separation of length scales where the surface roughness occurs on length scales much smaller than the macroscopic curvature radius of the bodies. In this case we can integrate out the short-range surface roughness and obtain an effective flow current, which only depends on the macroscopic shape of the pressure and interfacial separation. To do this we first write (5) as

$$
\mathbf{J}=-\frac{u^{3}}{12 \eta^{*}} \nabla p+\frac{1}{2} u \mathbf{v}_{0}^{*}
$$

where

$$
\begin{aligned}
\eta^{*} & =\frac{\bar{u}+l}{\bar{u}+4 l} \eta, \\
\mathbf{v}_{0}^{*} & =\frac{\bar{u}}{\bar{u}+l} \mathbf{v}_{0} .
\end{aligned}
$$

Note that $\eta^{*}$ and $\mathbf{v}_{0}^{*}$ can be treated as constants when integrating out the short-wavelength roughness. Hence we can follow the procedure described elsewhere (see ref. [25]) and obtain

$$
\overline{\mathbf{J}}=-\frac{\bar{u}^{3} \phi_{\mathrm{p}}}{12 \eta^{*}} \nabla \bar{p}+\frac{1}{2} \bar{u} \mathbf{v}_{0}^{*}+\frac{1}{2} h_{\mathrm{rms}} \phi_{\mathrm{s}} \mathbf{v}_{0}^{*}
$$

where the pressure flow factor $\phi_{\mathrm{p}}$ and the shear flow factor $\phi_{\mathrm{s}}$ depend on the interfacial separation $\bar{u}$.

Using the definitions (7) and (8) we get

$$
\overline{\mathbf{J}}=-\frac{\bar{u}^{3} \phi_{\mathrm{p}}^{\prime}}{12 \eta} \nabla \bar{p}+\frac{1}{2} \bar{u} \mathbf{v}_{0}+\frac{1}{2} h_{\mathrm{rms}} \phi_{\mathrm{s}}^{\prime} \mathbf{v}_{0},
$$

where

$$
\begin{aligned}
\phi_{\mathrm{p}}^{\prime} & =\frac{\bar{u}+4 l}{\bar{u}+l} \phi_{\mathrm{p}}, \\
\phi_{\mathrm{s}}^{\prime} & =\frac{\bar{u}}{\bar{u}+l}\left(\phi_{\mathrm{s}}-\frac{l}{h_{\mathrm{rms}}}\right),
\end{aligned}
$$

where $\phi_{\mathrm{p}}$ and $\phi_{\mathrm{s}}$ are given in ref. [25].

\subsection{Frictional shear stress}

The fluid frictional shear stress on the surface $z=0$ is

$$
\sigma_{z x}=-\eta \frac{\partial \mathbf{v}}{\partial z}=\frac{1}{2} \frac{u^{2}}{u+l} \nabla p+\frac{\eta}{u+l} \mathbf{v}_{0} .
$$

Thus

$$
\left\langle\sigma_{z x}\right\rangle=\frac{1}{2}\left\langle\frac{u^{2}}{u+l} \nabla p\right\rangle+\left\langle\frac{\eta}{u+l}\right\rangle \mathbf{v}_{0} .
$$

We write the ensemble averaged fluid shear stress as

$$
\left\langle\sigma_{z x}\right\rangle=\left(\phi_{\mathrm{f}}^{\prime}+\phi_{\mathrm{fs}}^{\prime}\right) \frac{\eta_{0} \mathbf{v}_{0}}{\bar{u}}+\frac{1}{2} \phi_{\mathrm{fp}}^{\prime} \bar{u} \nabla \bar{p}
$$

With this definition the friction factor

$$
\phi_{\mathrm{f}}^{\prime}=\frac{\bar{u}}{\eta_{0}}\left\langle\frac{\eta}{u+l}\right\rangle .
$$

The friction factors $\phi_{\mathrm{fs}}^{\prime}$ and $\phi_{\mathrm{fp}}^{\prime}$ can be determined approximately as in ref. [2]. Assume that the contact area percolates for the average interfacial separation $\bar{u}_{\mathrm{c}}$. When the contact area percolates (i.e., for $\bar{u}<\bar{u}_{\mathrm{c}}$ ) the flow current must vanish, $\mathbf{J}=\mathbf{0}$, so that from (4)

$$
\begin{aligned}
\left\langle\frac{u^{2}}{u+l} \nabla p\right\rangle= & 6\left\langle\frac{\eta u}{(u+4 l)(u+l)}\right\rangle \mathbf{v}_{0} \approx \\
& 6 \frac{\bar{u}}{\bar{u}+4 l}\left\langle\frac{\eta}{u+l}\right\rangle \mathbf{v}_{0}=6 \phi_{\mathrm{f}}^{\prime} \frac{\eta_{0}^{*}}{\bar{u}} \mathbf{v}_{0}^{*} .
\end{aligned}
$$

Let us now assume $\bar{u} \gg h_{\text {rms }}$ and let us approximate

$$
\left\langle\frac{u^{2}}{u+l} \nabla p\right\rangle \approx \frac{\bar{u}}{\bar{u}+l}\langle u \nabla p\rangle .
$$

For $\bar{u} \gg h_{\text {rms }}$ we can use the result for $\langle u \nabla p\rangle$ derived in ref. [2]. We get

$$
\begin{aligned}
\left\langle\frac{u^{2}}{u+l} \nabla p\right\rangle \approx & \frac{\bar{u}^{4}}{(\bar{u}+l)\left(\bar{u}^{2}+3 h_{\mathrm{rms}}^{2} D\right)} \nabla \bar{p} \\
& +\frac{6}{(\bar{u}+l) \bar{u}^{2}} h_{\mathrm{rms}}^{2} D \eta_{0}^{*} \mathbf{v}_{0}^{*} .
\end{aligned}
$$

We can smoothly interpolate between the result (17) and (18) using

$$
\begin{aligned}
\left\langle\frac{u^{2}}{u+l} \nabla p\right\rangle \approx & \frac{\bar{u}^{3}\left(\bar{u}-\bar{u}_{\mathrm{c}}\right) \theta\left(\bar{u}-\bar{u}_{\mathrm{c}}\right)}{(\bar{u}+l)\left(\bar{u}^{2}+3 h_{\mathrm{rms}}^{2} D\right)} \nabla \bar{p} \\
& +\frac{6 \eta_{0}^{*} \mathbf{v}_{0}^{*}}{\bar{u} / \phi_{\mathrm{f}}^{\prime}+\theta\left(\bar{u}-\bar{u}_{\mathrm{c}}\right)\left(\bar{u}-\bar{u}_{\mathrm{c}}\right) \bar{u}(\bar{u}+l) h_{\mathrm{rms}}^{-2} D^{-1}} .
\end{aligned}
$$

Thus

$$
\phi_{\mathrm{fs}}^{\prime}=\frac{3 \bar{u}^{2} /(\bar{u}+4 l)}{\bar{u} / \phi_{\mathrm{f}}^{\prime}+\theta\left(\bar{u}-\bar{u}_{\mathrm{c}}\right)\left(\bar{u}-\bar{u}_{\mathrm{c}}\right) \bar{u}(\bar{u}+l) h_{\mathrm{rms}}^{-2} D^{-1}}
$$

and

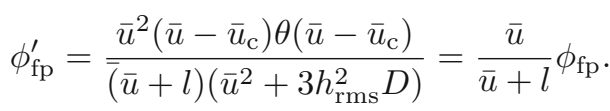




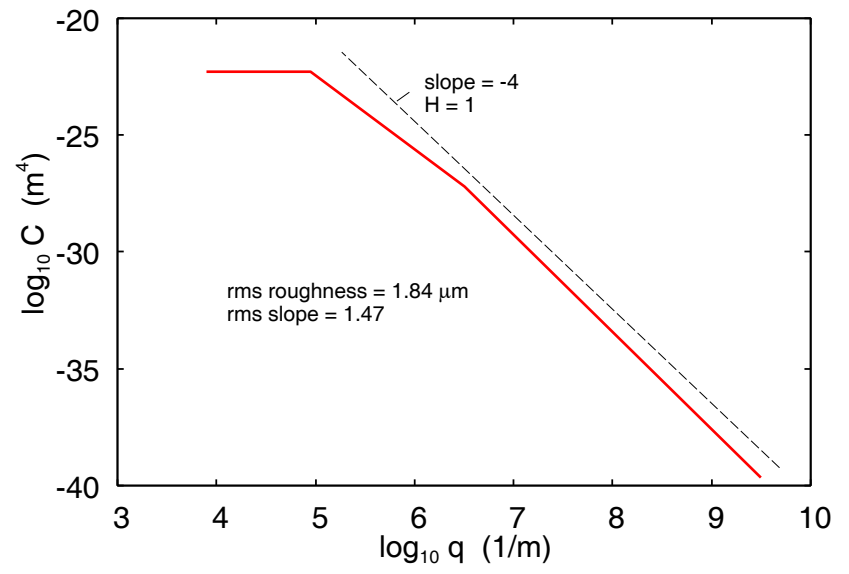

Fig. 4. The power spectrum used in the theory calculations. The dashed line has the slope -4 corresponding to the Hurst exponent $H=1$.

\section{Numerical results}

We consider the sliding of an elastic cylinder on a rigid lubricated flat surface. The flat (rigid) surface is assumed perfectly smooth, while the elastic cylinder has random roughness with the surface roughness power spectra shown in fig. 4. The surface roughness power spectrum is similar, but with larger rms roughness (by a factor of $\sim 3$ ), than that typically found for the rib of a syringe rubber stopper (see sect. 5.1). We use this larger surface roughness in this study since the smaller roughness results in some numerical convergence problems (see sect. 5.2).

The lubricant fluid in the following calculations is assumed to be a silicone oil with the small shear-rate viscosities $\eta_{0}=1,10$ and $100 \mathrm{~Pa}$ s. Silicone oils are known to shear thin at large enough shear rate $\dot{\gamma}$, and here we will use

$$
\eta=\frac{\eta_{0}}{1+\left(\eta_{0} / \eta_{1}\right)\left(\dot{\gamma} / \dot{\gamma}_{0}\right)^{\alpha}}
$$

with $\dot{\gamma}_{0}=1 \mathrm{~s}^{-1}, \eta_{1}=1.5 \times 10^{4} \mathrm{Pas}$ and $\alpha=0.77$, which gives good agreement with measured viscosity data for PDMS silicone oil $[26,27]$. Below we assume $\eta_{0}=1 \mathrm{Pas}$ unless otherwise stated.

\subsection{Fluid flow factors and friction factors}

Using the equations above, we have calculated the pressure and shear flow factors $\phi_{\mathrm{p}}^{\prime}$ and $\phi_{\mathrm{s}}^{\prime}$, and the friction factors $\phi_{\mathrm{f}}^{\prime}, \phi_{\mathrm{fp}}^{\prime}$ and $\phi_{\mathrm{fs}}^{\prime}$. In particular, the flow and friction factors given by (11), (12), (16), (20) and (21) depend on the average interfacial separation $\bar{u}$ which was derived in [28]. The friction factors $\phi_{\mathrm{f}}^{\prime}$ and $\phi_{\mathrm{fs}}^{\prime}$ depend also on the quantity

$$
\left\langle\frac{\eta}{u+l}\right\rangle=\int_{0}^{\infty} \mathrm{d} u \frac{\eta}{u+l} P(u)
$$

where $P(u)$ is the probability distribution of interfacial separations. This function was derived in ref. [29] (see also [30]).
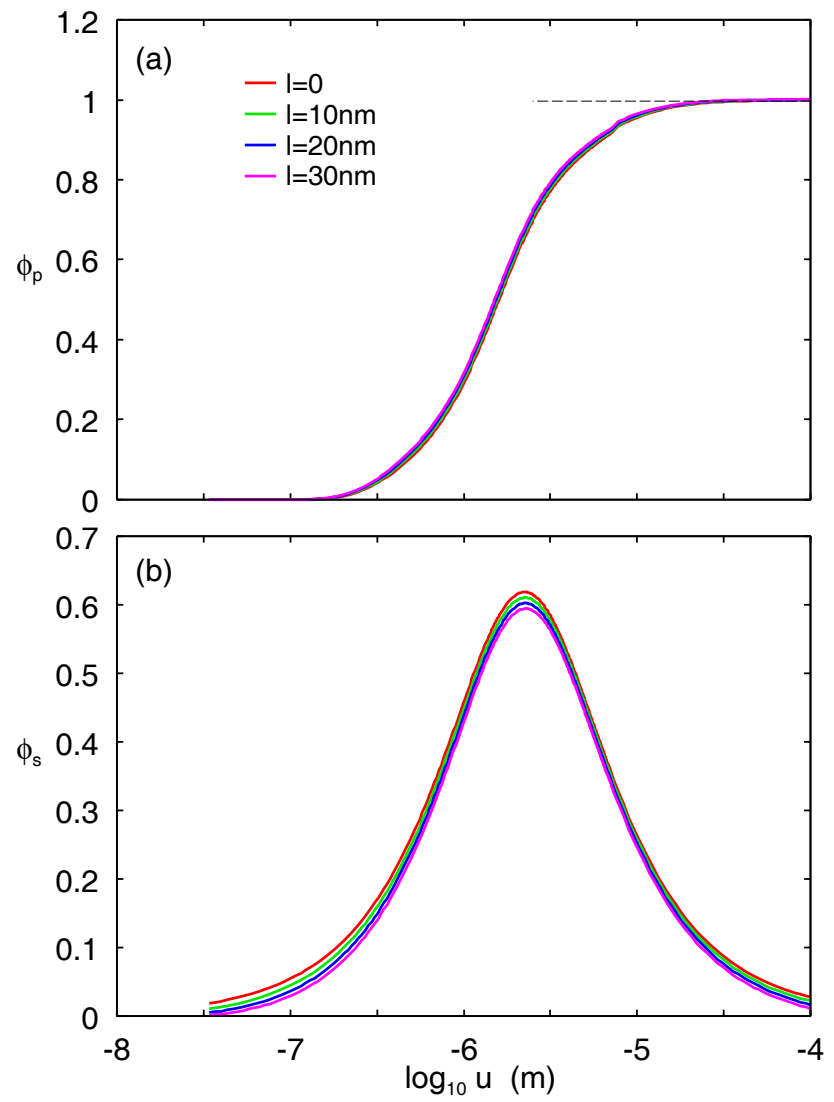

Fig. 5. Pressure and shear flow factors for the surface with the power spectrum given in fig. 4 .

In what follows we will, for simplicity, denote the flow and friction factors given by (11), (12), (16), (20) and (21) without the prime, i.e. by $\phi$ instead of $\phi^{\prime}$.

With the power spectrum given in fig. 4, we show results for the slip length $l=0$ (red), $l=10 \mathrm{~nm}$ (green), $l=20 \mathrm{~nm}$ (blue) and $l=30 \mathrm{~nm}$ (pink) in figs. 5 and 6 . Note that in the present case the slip has only a very small influence on the fluid pressure and shear flow factors. This imply that the dependence of the average interfacial separation, the fluid and solid (normal) contact pressures, and the area of (asperity) contact on the sliding speed are nearly independent of the slip boundary condition. However, the fluid slip has a drastic influence on the friction factors $\phi_{\mathrm{f}}$ and $\phi_{\mathrm{fs}}$ (see fig. 6(a) and (b)), and hence on the friction force. This can be easily understood as follows. Let us first consider $\phi_{\mathrm{f}}$ when the slip length $l=0$. In this case, if we consider for simplicity a Newtonian fluid

$$
\phi_{\mathrm{f}}=\bar{u}\left\langle\frac{1}{u}\right\rangle
$$

where $\langle\ldots\rangle$ stands for ensemble averaging, which in most cases can be replaced by averaging over the interfacial surface area. Consider the simplest case where the surface separation takes only two values, say $u_{1}$ and $u_{2}$ each in half of the total surface area (see fig. 7 ). In this case $\bar{u}=\langle u\rangle=$ $\left(u_{1}+u_{2}\right) / 2$, while $\left\langle u^{-1}\right\rangle=\left(1 / u_{1}+1 / u_{2}\right) / 2$. Thus, if $u_{1} \ll$ $u_{2}$ we get $\phi_{\mathrm{f}} \approx u_{2} / u_{1} \gg 1$. That is, $\phi_{\mathrm{f}}$ is dominated by 

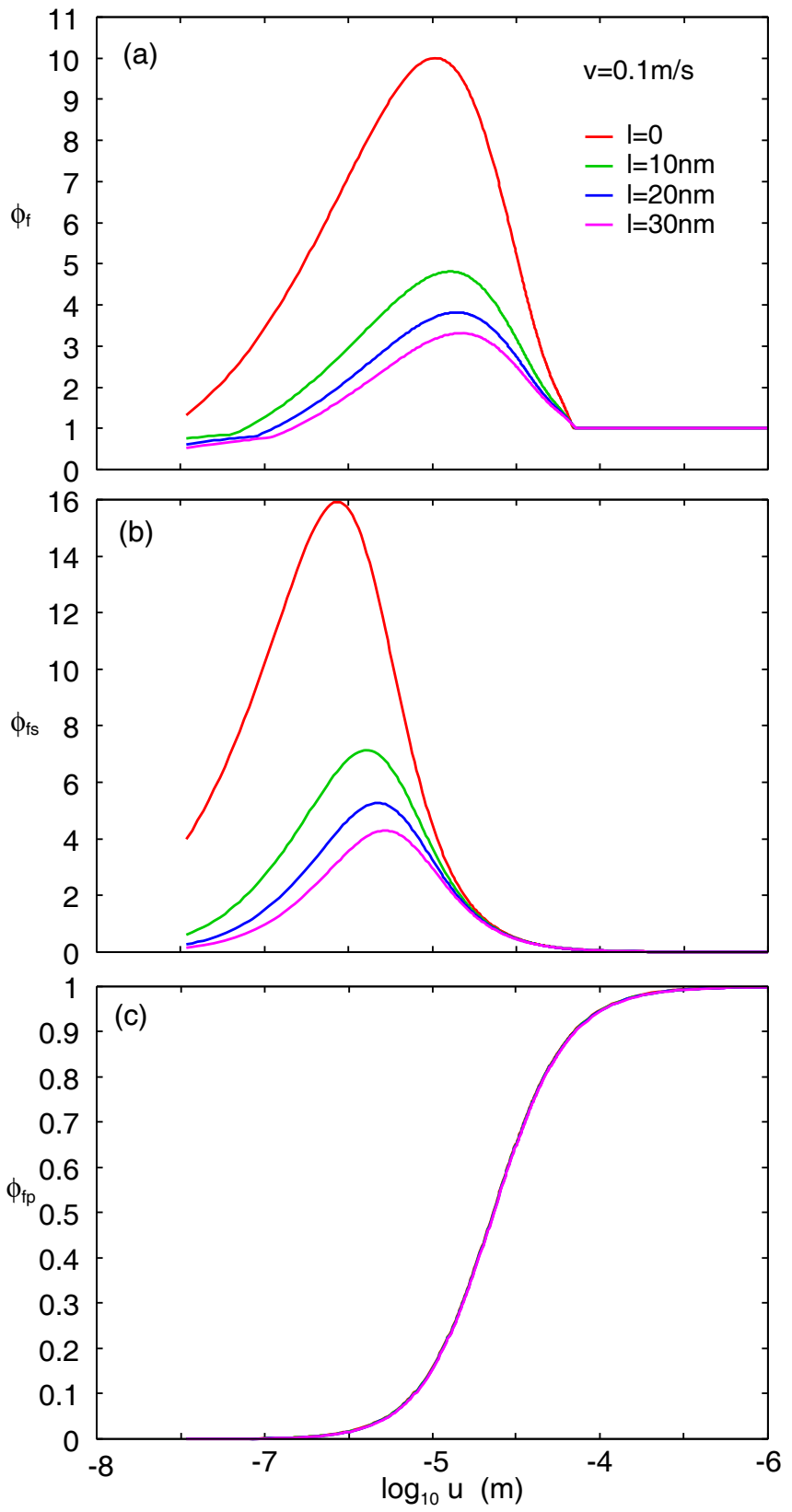

Fig. 6. Friction factors for the surface with the power spectrum given in fig. 4 . The friction factors $\phi_{\mathrm{f}}(\bar{u})$ and $\phi_{\mathrm{fs}}(\bar{u})$ are for the sliding speed $v=0.1 \mathrm{~mm} / \mathrm{s}$, while $\phi_{\mathrm{fp}}(\bar{u})$ is independent of $l$.

the surface area where the surface separation is very small, typically of order nanometer. Now consider the influence of the slip. In this case (assuming again a Newtonian fluid)

$$
\phi_{\mathrm{f}}=\bar{u}\left\langle\frac{1}{u+l}\right\rangle \text {. }
$$

For the case studied above, assuming $u_{1}+l \ll u_{2}+l$, we get $\phi_{\mathrm{f}} \approx u_{2} /\left(u_{1}+l\right)$. Now if $l$ is of order $u_{1}$ or larger, we obtain a large reduction in $\phi_{\mathrm{f}}$ when slip occurs. Note that $\phi_{\mathrm{fs}}$ depends on $\phi_{\mathrm{f}}$ so the same argument applies to $\phi_{\mathrm{fs}}$.

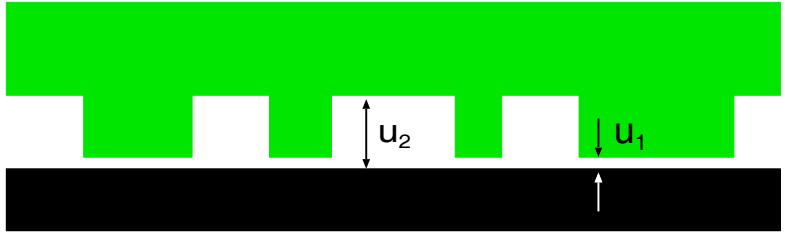

Fig. 7. The function $\bar{u}\left\langle u^{-1}\right\rangle$ is dominated by the contribution from the region where the interfacial separation $u$ is smallest (here $u=u_{1}$ ).

\subsection{Stribeck curve}

We consider the sliding of an elastic cylinder on a rigid lubricated flat surface. Young's elastic modulus of the cylinder is $E_{0}=2.5 \mathrm{MPa}$ and its radius of curvature is $R=0.4 \mathrm{~mm}$. The cylinder is squeezed against the flat surface with a force per unit length $F_{\mathrm{N}} / L=200 \mathrm{~N} / \mathrm{m}$. This results in a Hertz contact area of width $w=0.35 \mathrm{~mm}$. The maximum contact pressure is $0.73 \mathrm{MPa}$ and the average contact pressure is $0.57 \mathrm{MPa}$.

The elastic cylinder has random surface roughness with the surface roughness power spectrum given in fig. 4 . The cylinder is sliding with a constant speed $v_{0}$ in the $x$-direction (with the cylinder axis along the $y$-direction) on a perfectly flat and lubricated surface. If we consider the system in a reference frame where the cylinder is stationary, the fluid flow current satisfies $\mathrm{d} J_{x} / \mathrm{d} x=0$ or $J_{x}=v_{0} u^{*} / 2$ where $u^{*}$ is a constant. Using (10) we get

$$
\frac{\mathrm{d} \bar{p}}{\mathrm{~d} x}=\frac{6 \eta v_{0}}{\bar{u}^{3} \phi_{\mathrm{p}}(\bar{u})}\left(\bar{u}+h_{\mathrm{rms}} \phi_{\mathrm{s}}(\bar{u})-u^{*}\right) .
$$

The expression for the friction force (which depends on $\phi_{\mathrm{f}}(\bar{u}), \phi_{\mathrm{fp}}(\bar{u})$ and $\left.\phi_{\mathrm{fs}}(\bar{u})\right)$ is given by (15); see also refs. [2] and [3].

Hereinafter we will denote the (locally averaged) fluid pressure $\bar{p}$ as $p_{\text {fluid }}(\mathbf{x}, t)$. When solving for the fluid flow between macroscopic surfaces with roughness, in a meanfield type of treatment one writes the local nominal pressure (i.e., the pressure locally averaged over surface area with linear dimension of order the wavelength $\lambda_{0}$ of the longest surface roughness component) as

$$
p(\mathbf{x}, t)=p_{\text {fluid }}(\mathbf{x}, t)+p_{\text {con }}(\mathbf{x}, t)
$$

where $p_{\text {fluid }}$ and $p_{\text {con }}$ are the locally averaged nominal fluid pressure and solid wall-wall contact pressure, respectively. The fluid pressure satisfies (23), while the contact pressure $p_{\text {con }}$ can be related to the interfacial separation $\bar{u}(\mathbf{x}, t)$ using the contact mechanics theory of Persson (see refs. [2, 28-31]). In particular, for large enough average surface separation it can be shown that $p_{\text {con }} \approx \beta E \exp \left(-\bar{u} / u_{0}\right)$, where $\beta$ and $u_{0}$ can be calculated from the surface roughness power spectrum. We have solved (23) together with the equation for the macroscopic elastic deformations of the solids in response to the pressure $p(\mathbf{x}, t)$. For more details see refs. $[2,31]$.

Figure 8 shows the smallest interfacial surface separation $\bar{u}_{\text {min }}$ as a function of the sliding speed (log-log scale). 


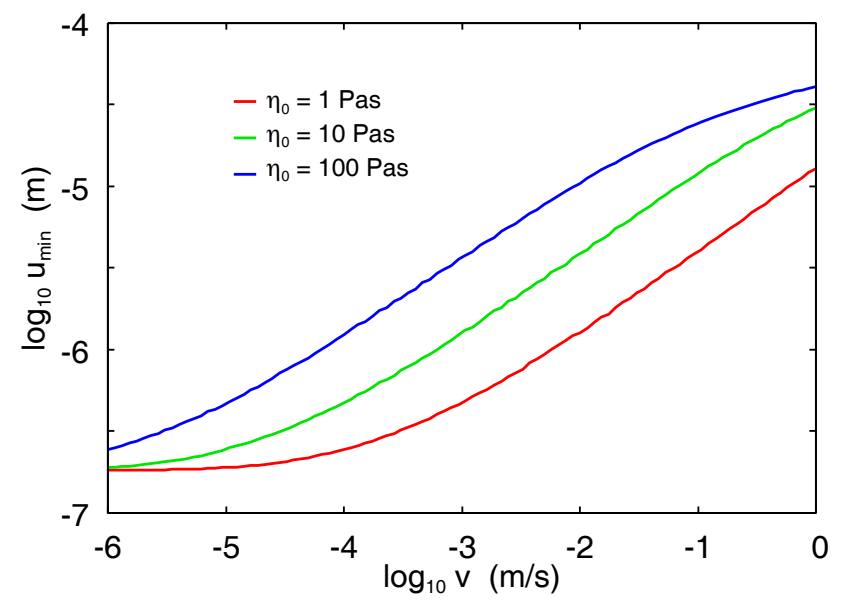

Fig. 8. The smallest interfacial separation $\bar{u}_{\text {min }}$ as a function of the sliding speed (log-log scale) for the (low strain-rate) fluid viscosity $\eta_{0}=1,10$ and $100 \mathrm{Pas}$.

In the calculations we have assumed no slip but, as pointed out above, the average interfacial separation depends very weakly on the slip boundary conditions so the results in fig. 8 are nearly the same for finite slip. For large sliding speeds hydrodynamic lubrication prevails, and here the average surface separation increases with increasing sliding speed because of the increase in the fluid pressure. As the sliding speed is reduced one enters into the boundary lubrication region where the external load is carried by the asperity contact regions. For $\eta_{0}=1 \mathrm{Pas}$ this occurs already for $v \approx 10^{-5} \mathrm{~m} / \mathrm{s}$ but for the fluids with higher viscosity, the boundary lubrication region occurs for lower velocities than considered in our study.

Figure 9 shows the kinetic friction coefficient as a function of the logarithm of the sliding velocity for the viscosity and slip length (a) $\eta_{0}=1 \mathrm{Pas}, l=10 \mathrm{~nm}$, (b) $\eta_{0}=10 \mathrm{Pas}, l=100 \mathrm{~nm}$, and (c) $\eta_{0}=100 \mathrm{Pas}$, $l=1000 \mathrm{~nm}$. The green lines are for $l=0$. Here we have assumed that the frictional shear stress in the rubber asperity contact regions is velocity independent and equal to $\tau_{\mathrm{f}}=0.04 \mathrm{MPa}$, as found for a rubber stopper on a glass surface with baked-on silicone oil (see sect. 5.2).

Figure 10 shows the kinetic friction coefficient as a function of the logarithm of the sliding velocity for the slip length $l=0,20 \mathrm{~nm}, 100 \mathrm{~nm}$ and $150 \mathrm{~nm}$ and with $\eta_{0}=0.35 \mathrm{Pas}$. Note that the friction coefficient is independent of the velocity for $v<10^{-5} \mathrm{~m} / \mathrm{s}$. This is the boundary lubrication region where the fluid hydrodynamic pressure is too small to affect the friction force. For the $\operatorname{slip} l=0$ there is a local maximum in $\mu_{\mathrm{k}}(v)$ at $v \approx 2 \mathrm{~cm} / \mathrm{s}$. This is due to shearing the lubrication film in the region where the average surface separation is very small and reflects the large magnitude of the friction factors $\phi_{\mathrm{f}}$ and $\phi_{\mathrm{fs}}$. Since the contribution from shearing the lubrication film in the region where the interfacial separation is very small is strongly reduced when the slip-length $l$ becomes large enough, this explains why this peak disappears when the slip length increases. This frictional shear flow peak (for $l=0$ ) has been observed in lubricated sliding fric-
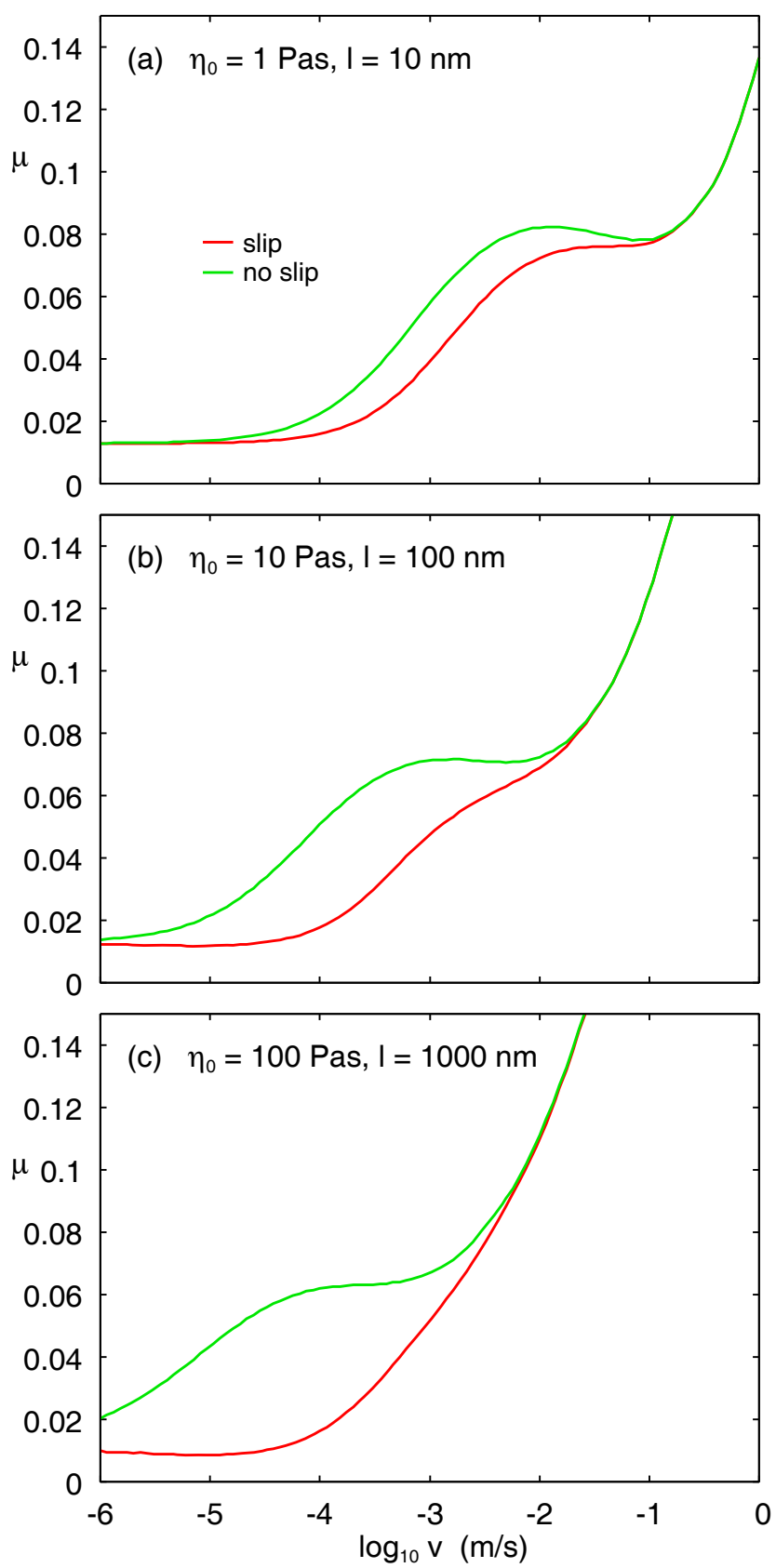

Fig. 9. The kinetic friction coefficient as a function of the logarithm of the sliding velocity. Results are shown for the viscosity and slip length (a) $\eta_{0}=1 \mathrm{Pas}, l=10 \mathrm{~nm}$, (b) $\eta_{0}=$ $10 \mathrm{Pas}, l=100 \mathrm{~nm}$, and (c) $\eta_{0}=100 \mathrm{Pas}, l=1000 \mathrm{~nm}$. The green lines are for $l=0$. We have used $\tau_{\mathrm{f}}=0.04 \mathrm{MPa}$.

tion experiments with glycerol as lubricant [2,32]. Note that glycerol is a Newtonian fluid up to large shear rates, which probably obeys the stick boundary conditions on the solid walls, i.e. $l \approx 0$.

Figure 9 shows that the friction curve $\mu(v)$ without slip (green curves) is shifted to smaller velocities as the viscosity increases. This is due to the increase in the frictional shear stress and the fluid pressure as the viscosity increases at a fixed sliding speed. The red curves includes 


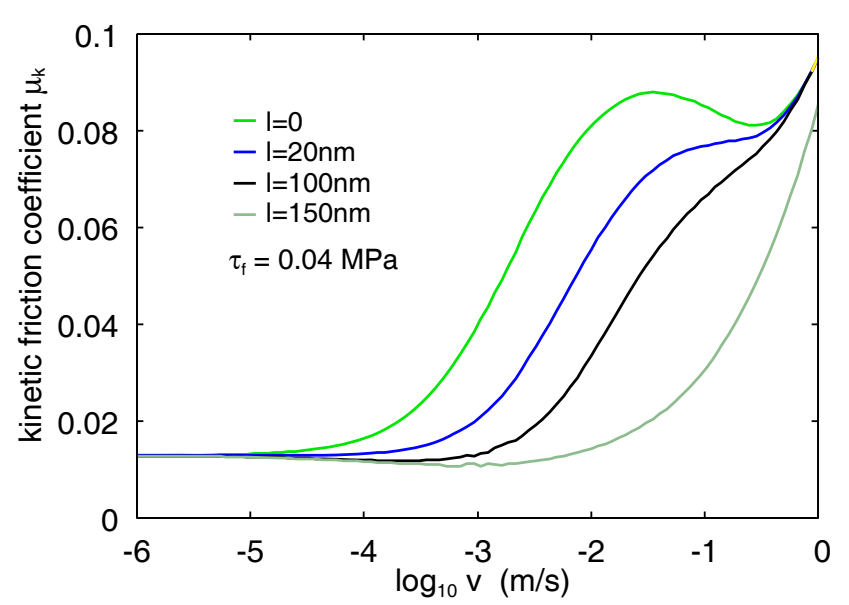

Fig. 10. The kinetic friction coefficient as a function of the logarithm of the sliding velocity. Results are shown for the slip lengths $l=0,20 \mathrm{~nm}, 100 \mathrm{~nm}$ and $150 \mathrm{~nm}$, and using $\eta_{0}=$ $0.35 \mathrm{Pas}$ and $\tau_{\mathrm{f}}=0.04 \mathrm{MPa}$.

slip, and we have assumed that the slip length is proportional to the viscosity as expected in the simplest theory description where $l=\eta / C$ (see sect. 1$)$. The increase in $l$ with increasing viscosity is the reason of the increased reduction in the frictional shear flow peak with increasing fluid viscosity.

\section{Sliding friction on glass surfaces with baked-on silicone oil}

Many applications require silica glass surfaces to exhibit very low friction (and low adhesion) against rubber. This can be realized by covering the glass surfaces with an inert coating. One important method is end-grafted short polydimethylsiloxan (PDMS) chains. Another very important method is baked-on siliconisation.

The siliconization of pharmaceutical glass primary containers such as syringes is either performed by spraying the silicone oil onto the inner surface of the container or by coating the glass with water-based silicone emulsion that is then baked on at high temperature. The use of the emulsion combined with the baking process allows obtaining a uniform and low friction silicone layer by using a reduced amount of lubricant with respect to the spraying process with only silicone oil.

Baked-on siliconisation involves the application of silicone oil (usually as an emulsion) on a glass surface, which then is baked on to the glass surface at a temperature of approximately $300^{\circ} \mathrm{C}$ for $10-30$ minutes inside a heating channel. The DOW CORNING® 365 siliconization emulsion, containing $35 \%$ of polydimethylsiloxane (PDMS), is often used in the baked-on siliconization process.

In the baked-on process, hydrogen and covalent bonds form between the glass surface and the polydimethylsiloxane chains (see fig. 11). This results in a very inert and hydrophobic coating, where the bonds are so strong that the attached polydimethylsiloxane chains cannot be removed with solvent [33].

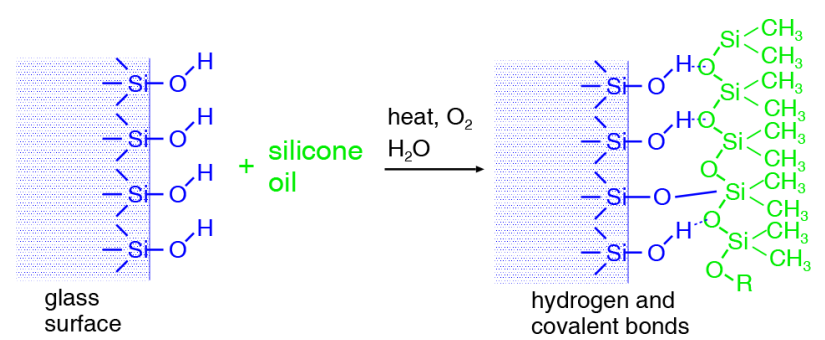

Fig. 11. Baked-on siliconisation involves the application of silicone oil (usually as an emulsion) on a glass surface, which then is baked on to the glass surface at a specific temperature and for a specific time period. In the baked-on process, hydrogen and covalent bonds form between the glass surface and the polydimethylsiloxane chains. This results in a very inert and hydrophobic coating, where the bonds are so strong that the attached polydimethylsiloxane chains cannot be removed with solvent.

The inert and stable nature of the baked-on silicone layer was shown in adhesion experiments [34]. Here a glass ball with baked-on silicone oil was moved into contact with a rubber sample (with a nominal flat surface) in a bath of silicone oil (viscosity $\eta=0.35 \mathrm{Pas}$ ). The ball was removed slowly $\left(v_{z} \approx 6 \mu \mathrm{m} / \mathrm{s}\right)$ after different waiting time periods. The pull-off (adhesion) force was found to be independent of the contact time for up to $\approx 1$ month contact time.

For the case of a clean glass ball the pull-off force was found to be much larger and to increase with increasing contact time. We interpret this as due to the squeeze-out of the silicone oil from the rubber-glass asperity contact regions, followed by bond formation between the rubber and the glass ball.

When prefilled syringes are stored for long time (which could be several years) the silicone oil is slowly squeezed out from the rubber-glass asperity contact regions. For glass surfaces without baked-on oil this may result in a very large breakloose friction force [35-37]. To lower the breakloose and sliding friction, glass barrels are often covered with baked-on silicone oil.

It is clear from the discussion above that during sliding on glass surfaces with baked-on silicon oil, slip may occur at the interface, which could result in a much lower sliding friction force than calculated using the stick boundary condition.

\subsection{Experimental: material and methods}

\section{Sample preparation}

In the experiments shown below, syringes are siliconized by using the baked-on method. To mimic conditions representatives of typical industrial processes, the silicone emulsion (35\% Dow Corning 365 Medical Fluid) is sprayed on the internal glass surface by using a pilot-scale siliconization unit (Bausch + Ströbel). Samples were then heated up to $T=300{ }^{\circ} \mathrm{C}$ for 20 minutes according to the standard industrial procedure. After baking, the containers are filled with water and closed with a rubber plunger stopper. 


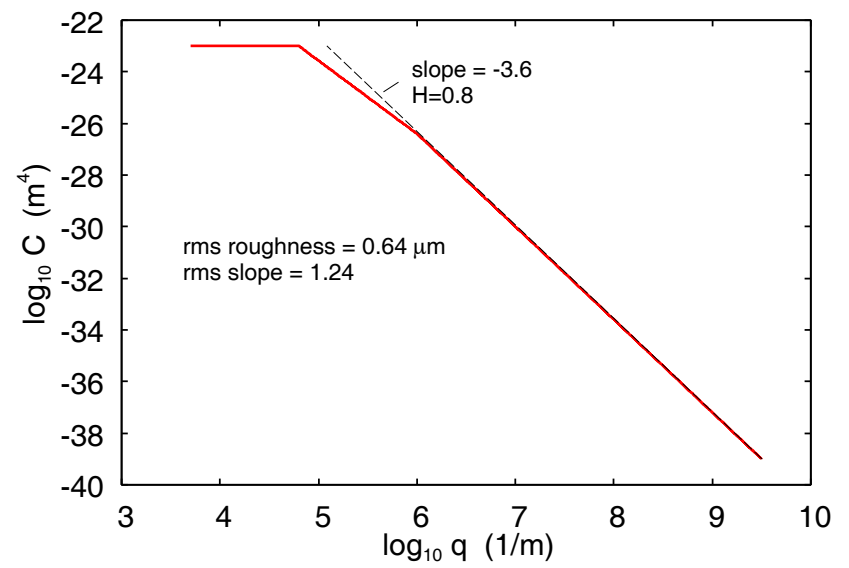

Fig. 12. Power spectrum of the rubber rib region of a rubber stopper. The dashed line has a slope equal to -3.6 corresponding to the Hurst exponent $H=0.8$.

\section{Measurement of the gliding force}

The measurement of the friction force of primary containers is performed in compression mode by using a Zwick bench Z5.0 (Zwick-Roell). The container is positioned in the sample holder, with the rubber plunger stopper upward. A plunger rod connected to a $50 \mathrm{~N}$ loading cell is gently placed in contact with the plunger stopper of the container then moved downward at constant speed. To generate a Stribeck curve, gliding tests were carried out at different speeds in the range from $1.2 \mathrm{~mm} / \mathrm{min}$ to $60 \mathrm{~mm} / \mathrm{min}$. Ten containers per speed were tested. During testing the force as a function of displacement is registered. Data are then analyzed to get the breakloose force value (not discussed in this paper), that is the force needed to start the movement of the rubber stopper and the gliding force, the force needed to expel the liquid from the container. Since the containers were needle free, no hydrodynamic force needs to be taken into account and the measured gliding force corresponds to the frictional force between the rubber stopper and the glass surface.

\section{Surface roughness power spectrum}

We focus on the friction force between a rubber stopper and a glass barrel lubricated by baked-on silicone oil. The glass barrel surface can be considered as perfectly smooth but the ribs of the rubber stopper, which are in contact with the glass surface, have a surface roughness which we have studied using an optical method, atomic force microscopy (AFM) and an engineering stylus instrument. From the measured surface topography we have calculated the surface roughness power spectrum $C(q)$ shown in fig. 12. The power spectrum is defined as

$$
C(q)=\frac{1}{(2 \pi)^{2}} \int \mathrm{d}^{2} x\langle h(\mathbf{x}) h(\mathbf{0})\rangle e^{i \mathbf{q} \cdot \mathbf{x}},
$$

where $h(\mathbf{x})$ is the height profile and $\langle\ldots\rangle$ stands for ensemble averaging. We have assumed $\langle h(\mathbf{x})\rangle=0$. For a
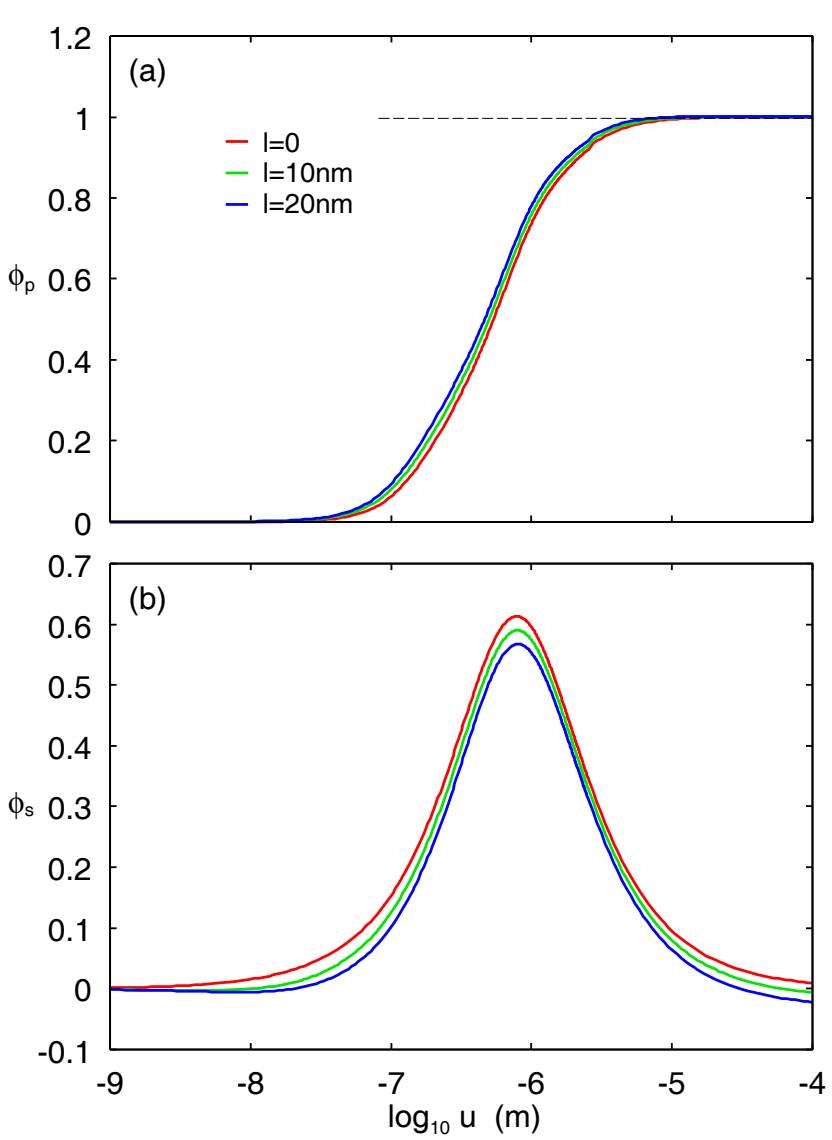

Fig. 13. Pressure and shear flow factors.

randomly rough surface, all (statistically averaged) information about the surface roughness is contained in the $C(q)$-function. Thus, for example, the root-mean-square $(\mathrm{rms})$ roughness and the rms slope are given as integrals of $C(q)$. In the present case the rms roughness is $0.64 \mu \mathrm{m}$ and the rms slope 1.24 .

\section{Fluid viscosity}

The lubricant fluid in the following calculations is assumed to be a silicone oil with the small shear-rate viscosity $\eta_{0}=$ 0.35 Pas. Silicone oils are known to shear thin at large enough shear rate, and here we will use $(22)$ with $\dot{\gamma}_{0}=$ $1 \mathrm{~s}^{-1}, \eta_{1}=1.5 \times 10^{4} \mathrm{Pas}$ and $\alpha=0.77$, which gives a viscosity in good agreement with the measured data for PDMS silicone oil.

\subsection{Numerical results and comparison with experiments}

\section{Fluid flow and friction factors}

Using the equations presented in sect. 3 we have calculated the pressure and shear flow factors $\phi_{\mathrm{p}}$ and $\phi_{\mathrm{s}}$, and the friction factors $\phi_{\mathrm{f}}, \phi_{\mathrm{fp}}$ and $\phi_{\mathrm{fs}}$. In figs. 13 and 14 we show the results for the slip length $l=0$ (red), $l=10 \mathrm{~nm}$ (green) 

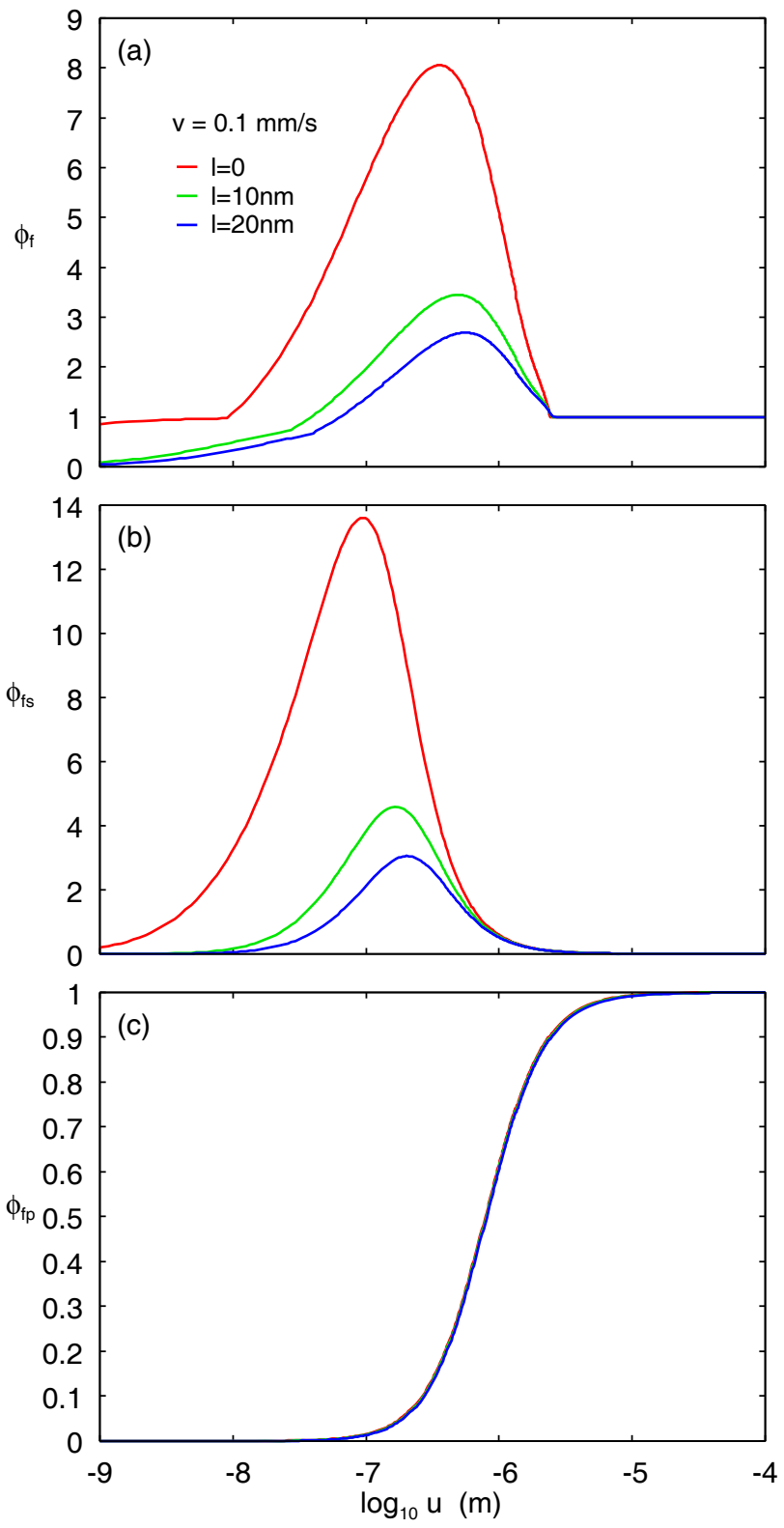

Fig. 14. Friction factors. The friction factors $\phi_{\mathrm{f}}(\bar{u})$ and $\phi_{\mathrm{fs}}(\bar{u})$ are for the sliding speed $v=0.1 \mathrm{~mm} / \mathrm{s}$, while $\phi_{\mathrm{fp}}(\bar{u})$ is independent of $v$.

and $l=20 \mathrm{~nm}$ (blue). The slip has only a rather small influence on the pressure and shear flow factors $\phi_{\mathrm{p}}$ and $\phi_{\mathrm{s}}$, which implies that the average interfacial separation, the asperity contact area, and the fluid and contact pressure distributions, are only weakly affected by the slip. However, the slip has a drastic influence on the friction factors $\phi_{\mathrm{f}}$ and $\phi_{\mathrm{fs}}$ (see fig. 14(a) and (b)), and hence on the friction force. The physical reason for this was discussed in sect. 4.1.

\section{Comparison with experiment}

We consider the sliding of an elastic cylinder on a rigid lubricated flat surface. The Young elastic modulus of the

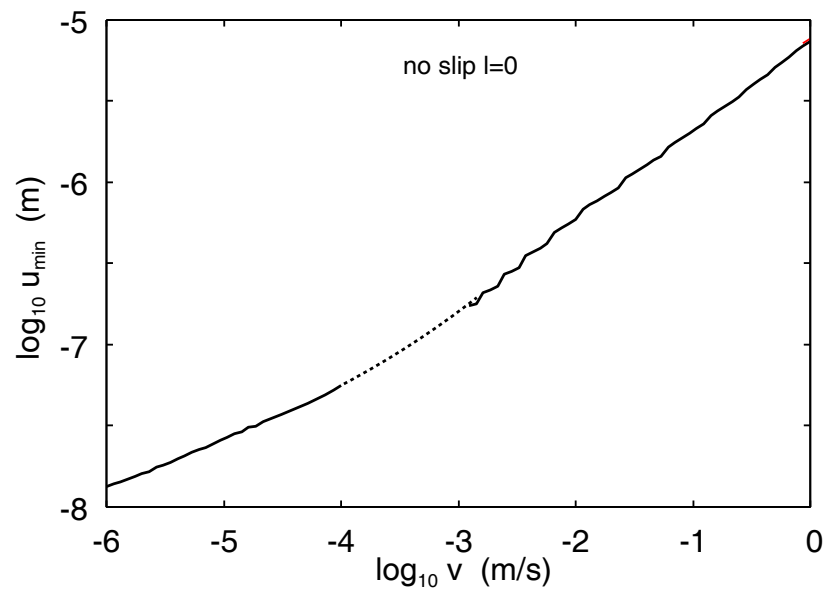

Fig. 15. The smallest interfacial separation $\bar{u}_{\min }$ as a function of the sliding speed (log-log scale).

cylinder is $E_{0}=2.5 \mathrm{MPa}$ and its radius of curvature is $R=$ $0.4 \mathrm{~mm}$. The cylinder is squeezed against the flat surface with a force per unit length $F_{\mathrm{N}} / L=200 \mathrm{~N} / \mathrm{m}$. This results in a Hertzian contact area of width $w=0.35 \mathrm{~mm}$. The maximum contact pressure is $0.73 \mathrm{MPa}$ and the average contact pressure is $0.57 \mathrm{MPa}$. The flat (rigid) surface is assumed perfectly smooth while the elastic cylinder has random roughness. The surface roughness power spectrum of the cylinder surface is given in fig. 12. In appendix A we show optical pictures when the rubber stopper is squeezed against a flat glass surface.

Consider now the elastic cylinder sliding with a constant speed $v_{0}$ in the $x$-direction (with the cylinder axis along the $y$-direction) on a perfectly flat and lubricated surface. We consider the system in a reference frame where the cylinder is not moving. The fluid pressure $\bar{p}(x)$ is given by (23) (see also ref. [2]). The expression for the friction force (which depends on $\phi_{\mathrm{f}}(\bar{u}), \phi_{\mathrm{fp}}(\bar{u})$ and $\left.\phi_{\mathrm{fs}}(\bar{u})\right)$ is given in sect. 3 (see also ref. [2]).

Figure 15 shows the smallest interfacial surface separation $\bar{u}_{\text {min }}$ as a function of the sliding speed (log-log scale). For large sliding speed the hydrodynamic lubrication prevails, but the boundary lubrication region (where $\bar{u}(x, y)$ is independent of the velocity), is not observed in the studied velocity range.

Because of the small (average) surface separation at low sliding speed $(\approx 10 \mathrm{~nm}$, at the lowest sliding speed $10^{-6} \mathrm{~m} / \mathrm{s}$ ) the mixed lubrication calculations becomes hard to converge for small velocities. In particular, there is a velocity region (dotted curve segment in fig. 15), from $\approx 10^{-4} \mathrm{~m} / \mathrm{s}$ to $\approx 10^{-3} \mathrm{~m} / \mathrm{s}$, where the simulations does not converge. However, this velocity region is small enough that we can smoothly interpolate to obtain the Stribeck curve also in this velocity interval.

Figure 16 shows the kinetic friction coefficient as a function of the logarithm of the sliding velocity for the rubber stopper in a glass barrel with baked-on silicone oil. The solid lines are the theory predictions for the slip lengths $l=0,10 \mathrm{~nm}$ and $20 \mathrm{~nm}$. In the calculation we have assumed that in the rubber-glass asperity contact 


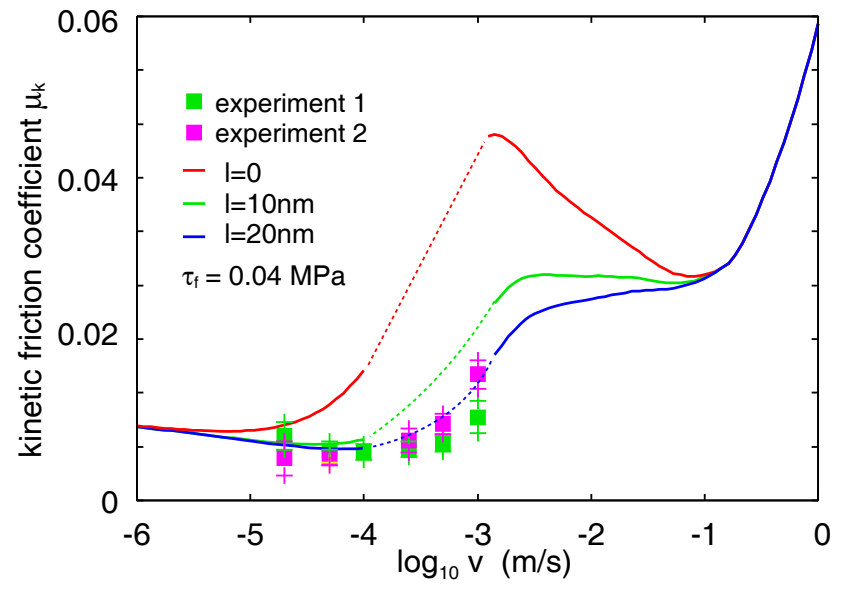

Fig. 16. The kinetic friction coefficient as a function of the logarithm of the sliding velocity for a rubber stopper in a glass barrel with baked-on silicone oil. The solid lines are the theory predictions for the slip lengths $l=0,10 \mathrm{~nm}$ and $20 \mathrm{~nm}$, and using $\tau_{\mathrm{f}}=0.04 \mathrm{MPa}$. The pink and green squares are measured data from two different set of syringes. The + symbols are the measured data with \pm standard deviation.

regions a (velocity independent) frictional shear stress $\tau_{\mathrm{f}}=0.04 \mathrm{MPa}$ acts. The pink and green squares are measured data from two different set of syringes. Note the good agreement between theory and experiment assuming the slip length $l=20 \mathrm{~nm}$, and that the friction coefficient varies rather weakly with the sliding speed for $v<10^{-4} \mathrm{~m} / \mathrm{s}$. We note that even if $\tau_{\mathrm{f}}=0$ the calculated friction coefficient without $\operatorname{sip}(l=0)$ would be too large for $v>10^{-4} \mathrm{~m} / \mathrm{s}$, i.e., it is impossible to explain the observed results assuming the no-slip fluid boundary condition.

We have also studied the kinetic friction for the case of not baked-on silicone oil [38]. In this case the friction at low sliding speed is much larger than for the baked-on oil film, and the frictional shear stress in the area of real contact is strongly velocity dependent (it decreases with increasing velocity in the velocity interval $10-1000 \mu \mathrm{m} / \mathrm{s}$ ), as expected from theory if some adhesive process, e.g. involving chain interdiffusion, operates in the area of real contact in the studied sliding velocity range.

\section{Summary and conclusion}

In studies of fluid flow between solids it is usually assumed that there is no slip between the fluid and solid walls. However in some applications, e.g., involving polymer fluids such as silicone oils, this assumption no longer holds. Here we have derived basic equations for the fluid flow between elastic solids with randomly rough surfaces including interfacial fluid slip. We have presented numerical results for realistic systems. In particular, we have considered an elastic cylinder with random surface roughness sliding on a flat rigid (low-energy) counter-surface (e.g., a glass surface with baked-on silicone oil) in a shear-thinning fluid such as silicone oil. The fluid slip, if large enough, will remove the frictional shear flow peak which otherwise would occur in the Stribeck curve in the mixed lubrication transition region.

We have also measured the kinetic friction force when (syringe) rubber ribs are sliding on glass surfaces lubricated with baked-on silicone oil. The experimental results can only be explained if slip occurs between the lubricant fluid and the baked-on oil film. Good agreement between the theory predictions and experimental data is obtained assuming a velocity-independent frictional shear stress in the area or real contact, and that the slip length is $l \approx 20 \mathrm{~nm}$.

Open Access Funding provided by Projekt DEAL. We thank Alexander Kovalev and Stanislav Gorb (Kiel University), and Enrico Gnecco (Friedrich Schiller University Jena), and Julia Persson (MultiscaleConsulting) for optical, AFM, and engineering stylus measurements of the surface topography of the rubber stopper. We wish to tank Marielle Dufraigne (PCDMED Vitry, Sanofi) for the preparation of not-baked samples and gliding force measurements and Michel Stroh (PCD-MED Frankfurt, Sanofi) for providing the backed-on samples. We also thank Bernd Kuehn (PCD-MED Frankfurt, Sanofi) for financial support. This work was performed within a ReinhartKoselleck project funded by the Deutsche Forschungsgemeinschaft (DFG). BNJP would like to thank DFG for the project support under the reference German Research Foundation DFG-Grant: MU 1225/36-1. BNJP also acknowledges supported by the DFG-grant: PE 807/12-1. MS would like to thank MIUR for the PRIN 2017 project support under the grant 2017948FEN (FASTire).

\section{Author contribution statement}

CR has performed the friction experiments reported on in fig. 16. BNJP has developed the theory (sect. 3), implemented it in the numerical code used to calculate the fluid flow and friction factors, and performed the numerical calculations. MS has implemented the fluid flow and friction factors in the mixed lubrication code. PM has contributed in the design of the research project. All authors have discussed the results, and contributed to writing-up the paper.

Publisher's Note The EPJ Publishers remain neutral with regard to jurisdictional claims in published maps and institutional affiliations.

\section{Appendix A. Dry contact}

The area of real contact and the average interfacial separation are two quantities of great importance for lubricated sliding friction. Here we consider these quantities for dry (no lubricant) stationary contact. It is crucial to understand that unless the squeezing pressure is so large that 


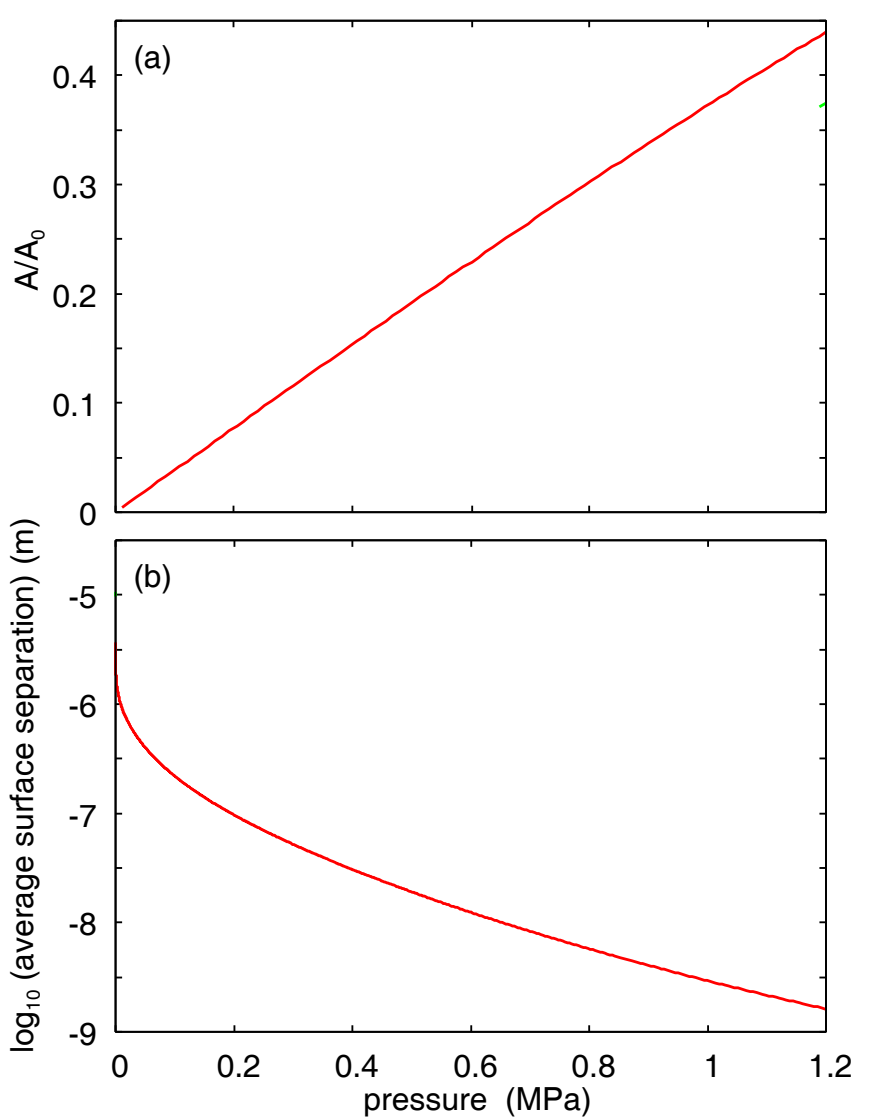

Fig. 17. The relative contact area $A / A_{0}$ and the logarithm of the average separation between the surfaces as a function of the applied pressure.

the contact is nearly complete, the average interfacial separation is mainly determined by the long-wavelength part of the surface roughness. On the other hand, the area of real contact depends on all surface roughness components, with the short-wavelength part which is particularly important. In fact, for randomly rough surfaces the contact area is determined by the surface rms slope. Figure 17(a) shows the relative contact area $A / A_{0}$ as a function of the squeezing pressure. Note that the relative contact area when $p \approx 1 \mathrm{MPa}$ is $A / A_{0} \approx 0.3$.

The average surface separation is determined mainly by the long-wavelength roughness. In fig. 17(b) we show the average surface separation as a function of the applied pressure. The average surface separation has a big influence on mixed lubrication (and fluid squeeze-out).

Finally, we show the optical picture of the contact between a rubber stopper and a smooth glass surface. Figure 18 shows the contact as the load decreases from (a) $F_{\mathrm{N}}=10 \mathrm{~N}$, (b) $6.7 \mathrm{~N}$ and (c) $3.3 \mathrm{~N}$. Note that at this low magnification it appears as if there is perfect contact within the nominal contact region. However, increasing the magnification shows that there is incomplete contact within the nominal contact region. This is illustrated in fig. 19 which shows the contact at two higher magnifications. The normal load acting is $F_{\mathrm{N}}=10 \mathrm{~N}$, and the
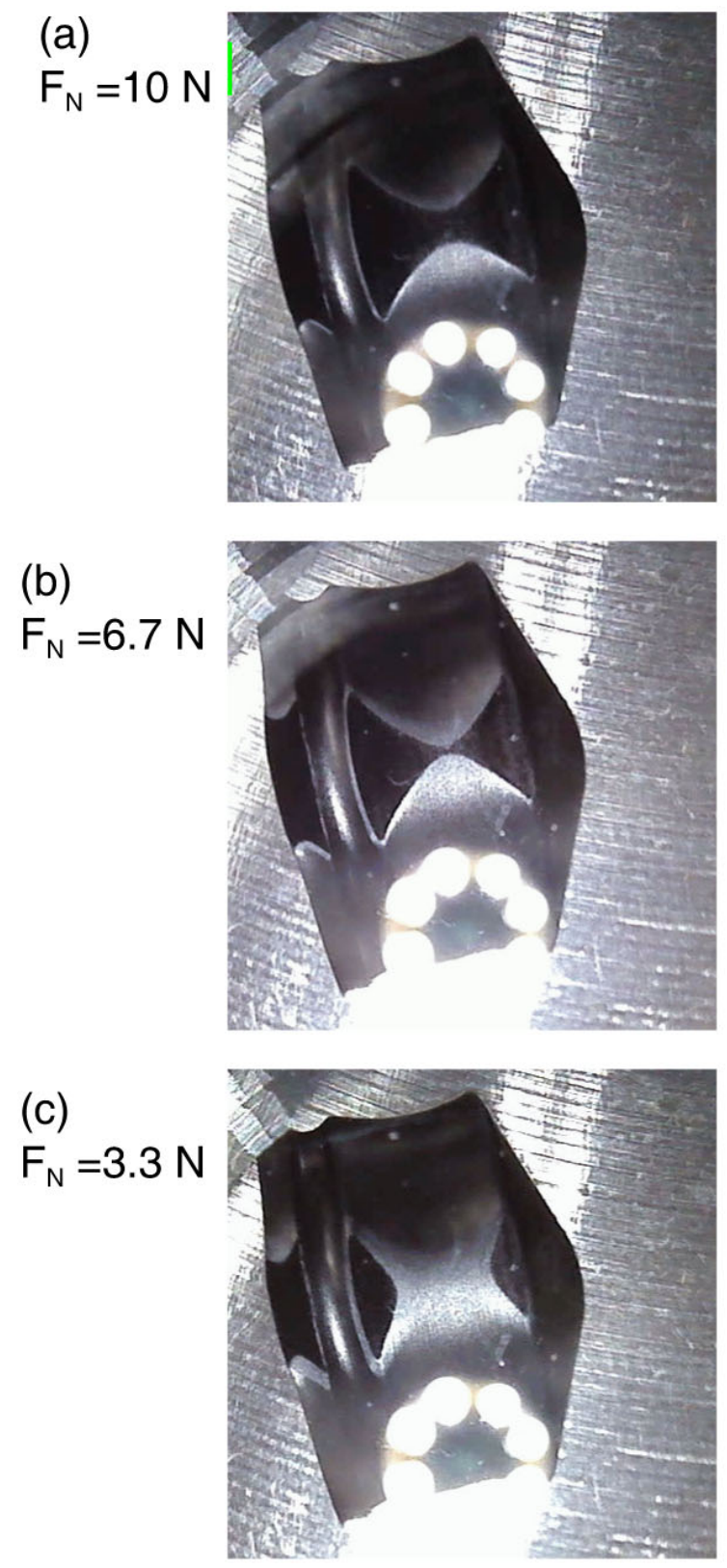

Fig. 18. Optical pictures of the contact between a rubber stopper and a flat glass surface at the normal load (a) $F_{\mathrm{N}}=$ $10 \mathrm{~N}$, (b) $6.7 \mathrm{~N}$ and (c) $3.3 \mathrm{~N}$. The width of the rubber stopper is about $12 \mathrm{~mm}$.

nominal contact pressure in the nominal contact area is about $p \approx 1 \mathrm{MPa}$. The pictures indicate a relative contact area $A / A_{0} \approx 0.5$ consistent with our theory calculations. However, it is not possible from optical pictures like fig. 19 to accurately define the area of contact as it depends on the gray-scale cut-off used to separate contact from noncontact. In addition, optical pictures never have atomic resolution, but rather the highest resolution is of order the wavelength of light (typically $\sim 1 \mu \mathrm{m}$ ). As the magnification increases the (apparent) area of contact continuously decreases. 

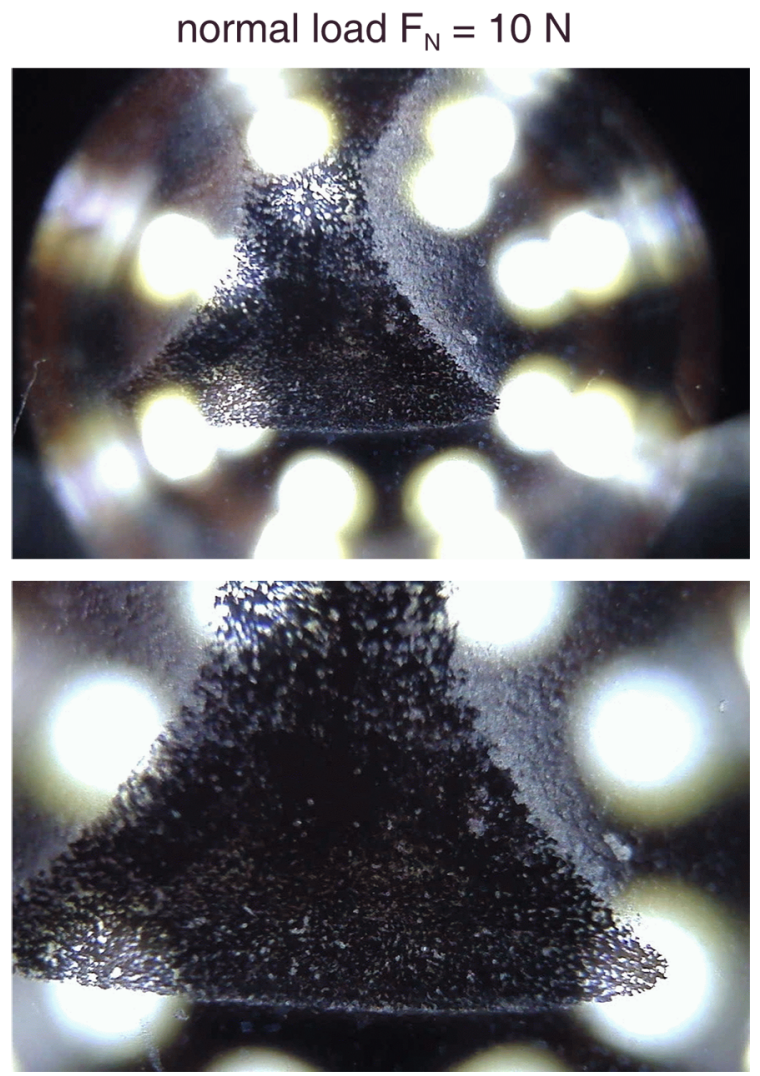

Fig. 19. Magnified view of the contact between a rubber stopper and a flat glass surface at the normal load $F_{\mathrm{N}}=10 \mathrm{~N}$.

Open Access This is an open access article distributed under the terms of the Creative Commons Attribution License (http://creativecommons.org/licenses/by/4.0), which permits unrestricted use, distribution, and reproduction in any medium, provided the original work is properly cited.

\section{References}

1. B. Hamrock, S. Schmid, B. Jacobson, Fundamentals of Fluid Film Lubrication, 2nd edition (CRC Press, 2004).

2. B.N.J. Persson, M. Scaraggi, Eur. Phys. J. E 34, 113 (2011).

3. M. Scaraggi, J. Angerhausen, L. Dorogin, H. Murrenhoff, B.N.J. Persson, Wear 410-411, 43 (2018).

4. G. de Boer, A. Almqvist, Lubricants 6, 78 (2018).

5. M. Söderfjäll, R. Larsson, P. Marklund, A. Almqvist, Proc. Inst. Mech. Eng. Part J: J. Eng. Tribol. 232, 166 (2018).

6. J. Krim, D.H. Solina, R. Chiarello, Phys. Rev. Lett. 66 181 (1991).
7. T. Coffey, J. Krim, Phys. Rev. Lett. 95, 076101 (2005).

8. J. Krim, Adv. Phys. 61, 155 (2012).

9. P.G. de Gennes, C.R. Acad. Sci. Paris B 288, 219 (1979).

10. B.N.J. Persson, J. Chem. Phys. 103, 3849 (1995).

11. B.N.J. Persson, Sliding Friction: Physical Principles and Applications (Springer, Heidelberg, 2000).

12. L. Leger, H. Hervet, G. Massey, E. Durliat, J. Phys.: Condens. Matter 9, 7719 (1997).

13. V. Mhetar, L.A. Archer, Macromolecules 31, 8607 (1998).

14. G. Massey, H. Hervet, L. Leger, Europhys. Lett. 43, 83 (1998).

15. R. Fetzer, M. Rauscher, A. Münch, B. Wagner, K. Jacobs, Europhys. Lett. 75, 638 (2006).

16. F. Brochard, P.G. De Gennes, Langmuir 8, 3033 (1992).

17. K.B. Migler, H. Hervet, L. Leger, Phys. Rev. Lett. 70, 287 (1993).

18. F. Brochard-Wyart, C. Gay, P.G. de Gennes, Macromolecules 29, 377 (1996).

19. E. Durliat, H. Hervet, L. Leger, Europhys. Lett. 38, 383 (1997).

20. S.Q. Wang, P.P. Drda, Macromol. Chem. Phys. 198, 673 (1997).

21. M. Henot, E. Drockenmuller, L. Leger $\dagger$, F. Restagno, ACS Macro Lett. 7, 112 (2018).

22. S. de Beer, M.H. Mueser, Macromolecules 47, 7666 (2014).

23. L.D. Landau, E.M. Lifshitz, Fluid Mechanics (Pergamon Press, 1975).

24. N. Patir, H.S. Cheng, J. Lubr. Technol. 100, 12 (1978).

25. B.N.J. Persson, J. Phys.: Condens. Matter 22, 265004 (2010).

26. D. Hadjistamov, J. Appl. Polym. Sci. 108, 2356 (2008).

27. C.L. Lee, K.E. Polmanteer, E.G. King, J. Polym. Sci. Part A-2: Polym. Phys. 8, 1909 (1970).

28. C. Yang, B.N.J. Persson, J. Phys.: Condens. Matter 20, 215214 (2008).

29. A. Almqvist, C. Campana, N. Prodanov, B.N.J. Persson, J. Mech. Phys. Solids 59, 2355 (2011).

30. L. Afferrante, F. Bottiglione, C. Putignano, B.N.J. Persson, G. Carbone, Tribol. Lett. 66, 75 (2018).

31. B.N.J. Persson, M. Scaraggi, J. Phys.: Condens. Matter 21, 185002 (2009).

32. M. Scaraggi, G. Carbone, D. Dini, Tribol. Lett. 43, 169 (2011).

33. B. Reuter, C. Petersen, TechnoPharm 2, 238 (2012).

34. L. Dorogin, A. Tiwari, C. Rotella, P. Mangiagalli, B.N.J. Persson, J. Chem. Phys. 148, 234702 (2018).

35. B. Lorenz, B.A. Krick, N. Rodriguez, Sawyer, P. Mangiagalli, B.N.J. Persson, J. Phys.: Condens. Matter 25, 445013 (2013).

36. B.N.J. Persson, N. Prodanov, B.A. Krick, N. Rodriguez, N. Mulakaluri, W.G. Sawyer, P. Mangiagalli, Eur. Phys. J. E 35, 5 (2012).

37. M. Scaraggi, B.N.J. Persson, Tribol. Lett. 47, 409 (2012). 38. C. Rotella, B.N.J. Persson, P. Mangiagalli, in preparation. 